\title{
Off-label use of combined antiretroviral therapy, analysis of data collected by The Italian Register for HIV-1 infection in Paediatrics in a large cohort of children.
}

\section{Elena Chiappini ( $\nabla$ elena.chiappini@unifi.it)}

Paediatric Infectious Diseases Unit, Department of Health Sciences, Anna Meyer Children University Hospital, Florence, Italy

Catiuscia Lisi

Paediatric Infectious Diseases Unit, Department of Health Sciences, Anna Meyer Children University Hospital, Florence, Italy

Vania Giacomet

Paediatric Infectious Diseases Unit, Department of Paediatrics, ASST FBF SACCO, University of Milan, Milan, Italy

\section{Paola Erba}

Paediatric Infectious Diseases Unit, Department of Paediatrics, ASST FBF SACCO, University of Milan, Milan, Italy

\section{Stefania Bernardi}

Department of Paediatric and Immunology “Bambino Gesù,” University Children’s Hospital, Rome, Italy

\section{Paola Zangari}

Department of Paediatric and Immunology “Bambino Gesù,” University Children's Hospital, Rome, Italy

\section{Antonio Di Biagio}

Infectious Disease Unit, Ospedale Policlinico San Martino, University of Genova, Genova, Italy

\section{Lucia Taramasso}

Infectious Disease Unit, Ospedale Policlinico San Martino, University of Genova, Genova, Italy

\section{Carlo Giaquinto}

Department of Women and Child Health, University of Padova, Padova, Italy

\section{Osvalda Rampon}

Department of Women and Child Health, University of Padova, Padova, Italy

\section{Clara Gabiano}

Paediatric Infectious Diseases Unit, Regina Margherita Children's Hospital, University of Turin, Turin, Italy

\section{Silvia Garazzino}

Paediatric Infectious Diseases Unit, Regina Margherita Children's Hospital, University of Turin, Turin, Italy

\section{Claudia Tagliabue}

Fondazione IRCCS Ca' Granda Ospedale Maggiore Policlinico

\section{Susanna Esposito}

Paediatric Clinic, Pietro Barilla Children's Hospital, University of Parma, Parma, Italy

\section{Eugenia Bruzzese}

Department of Translational Medical Sciences, Section of Paediatrics, University of Naples Federico II, Naples, Italy

\section{Raffaele Badolato}

Department of Clinical and Experimental Sciences, University of Brescia, Brescia, Italy

\section{Domenico Zanaboni}

Department on Internal Medicine and Therapeutics, IRCCS Policlinico "S. Matteo" Foundation, University of Pavia, Pavia, Italy

\section{Monica Cellini}

Department of Medical and Surgical Sciences, Section of Paediatric Hemato-Oncology, University of Modena and Reggio Emilia, Modena, Italy 


\section{Maurizio Dedoni}

Department of Paediatrics, Ospedale Microcitemico, Cagliari, Italy

\section{Antonio Mazza}

Paediatric Unit, "S. Chiara" Hospital, Trento, Italy

\section{Andrea Pession}

Paediatric Unit, Scientific Institute for Research and Healthcare (IRCCS), Sant'Orsola Hospital, Bologna, Italy

\section{Anna Maria Giannini}

Paediatric Infectious Diseases Unit, University Hospital Policlinico Giovanni XXIII, Bari, Italy

\section{Filippo Salvini}

Department of Paediatrics, University of Milan, Niguarda Hospital, Milan, Italy

\section{Icilio Dodi}

Paediatric Unit, Pietro Barilla Children's Hospital University of Parma, Parma, Italy

\section{Ines Carloni}

Department of Mother and Child Health, Salesi Children's Hospital, Ancona, Italy

\section{Salvatore Cazzato}

Department of Mother and Child Health, Salesi Children's Hospital, Ancona, Italy

\section{Pier Angelo Tovo}

Paediatric Infectious Diseases Unit, Regina Margherita Children's Hospital, University of Turin, Turin, Italy

\section{Maurizio de Martino}

Paediatric Infectious Diseases Unit, Department of Health Sciences, Anna Meyer Children University Hospital, Florence, Italy

\section{Luisa Galli}

Paediatric Infectious Diseases Unit, Department of Health Sciences, Anna Meyer Children University Hospital, Florence, Italy Italian Register for HIV Infection in Children Italy

Paediatric Infectious Diseases Unit, Department of Health Sciences, Anna Meyer Children University Hospital, Florence, Italy

\section{Research Article}

Keywords: HIV-1 infection, children, antiretroviral therapy, HAART, off-label therapy

Posted Date: March 19th, 2021

DOI: https://doi.org/10.21203/rs.3.rs-319571/v1

License: (c) (i) This work is licensed under a Creative Commons Attribution 4.0 International License. Read Full License

Version of Record: A version of this preprint was published at BMC Infectious Diseases on January 15th, 2022. See the published version at https://doi.org/10.1186/s12879-022-07026-w. 


\section{Abstract}

\section{Background}

Early start of highly active antiretroviral therapy (HAART) in perinatally HIV-1 infected children is the optimal strategy to prevent immunological and clinical deterioration. To date, according to EMA, only 35\% of antiretroviral drugs are licenced in children $<2$ years of age and $60 \%$ in those aged 2-12 years, due to the lack of adequate paediatric clinical studies on pharmacokinetics, pharmacodynamics and drug safety in children.

Methods

An observational retrospective study investigating the rate and the outcomes of off-label prescription of HAART was conducted on 225 perinatally HIV-1 infected children enrolled in the Italian Register for HIV Infection in Children and followedup from 2001 to 2018.

Results

$22.2 \%(50 / 225)$ of included children were receiving an off-label HAART regimen at last check. Only $26 \%(13 / 50)$ of off-label children had an undetectable viral load $(V L)$ before the commencing of the regimen and the $52.0 \%(26 / 50)$ had a CD $4+T$ lymphocyte percentage $>25 \%$. At last check, during the off label regimen, the $80 \%$ (40/50) of patients had an undetectable VL, and $90 \%$ (45/50) of them displayed CD4 + T lymphocyte percentage $>25 \%$. The most widely used off-label drugs were: dolutegravir/abacavir/lamivudine (16\%; 8/50), emtricitbine/tenofovir disoproxil (22\%; 11/50), lopinavir/ritonavir (20\%; 10/50) and elvitegravir/cobicistat/emtricitabine/ tenofovir alafenamide $(10 \% ; 10 / 50)$. At logistic regression analysis, detectable VL before starting the current HAART regimen was a risk factor for receiving an off-label therapy (OR:2.41; 95\%IC 1.13-5.19; P = 0.024). Moreover, children < 2 years of age were at increased risk for receiving off-label HAART with respect to older children (OR:3.24; 95\%IC $1063-7.3 ; P=0.001$ ). Even if our safety data regarding off-label regimens where poor, no adverse event was reported.

Conclusion

The prescription of an off-label HAART regimen in perinatally HIV-1 infected children was common, in particular in children with detectable VL despite previous HAART and in younger children, especially those receiving their first regimen. Our data suggest similar proportions of virological and immunological successes at last check among children receiving off-label or on-label HAART. Larger studies are needed to better clarify efficacy and safety of off-label HAART regimens in children, in order to allow the enlargement of on-label prescription in children.

\section{Introduction}

Over the years, a marked evolution of antiretroviral therapy has occurred; starting from 1987 with the introduction of the first antiretroviral (ARV) drug, zidovudine, to 1997 with the introduction of protease inhibitors, up to the most recent ARV classes including fusion inhibitors, integrase inhibitors and CCR5 inhibitors [1]. The progression of combination antiretroviral therapy (CART) has made possible for HIV-1 infection to become a chronic disease. On the other hand, the therapy has become more difficult to manage, especially in children. In these patients, potential issues concern the management of the cART, including poor adherence (especially in adolescents), the high burden of daily pills, the few age-appropriate drug formulations and the differences in pharmacokinetics between children and adults that contribute in determining the effectiveness and safety of the cART itself [2]. According to recent data, nowadays about $12 \%$ of cART treated children still experience treatment failure, mainly due to drug resistance or poor adherence to therapy, related to lack of paediatric formulations or poor palatability of liquid formulations [2]. Childhood is also associated with metabolic and pharmacokinetic changes, such as differences in absorption, distribution and elimination of drugs, reduced intestinal motility or slowed gastric emptying [3]. In adolescents, drug interactions (such as with oral contraceptives) and puberty, in addition to weight and age, could influence the dosage of drugs [4]. 
To date, only $34.4 \%$ of ARVs administered in adults are approved for use in children under 2 years of age, and $62 \%$ are approved in those under 12 years of age in Europe [5]. The approval for paediatric use of ARVs may be adventurous with variable latency of time compared to the adult. As an example, tenofovir disoproxil fumarate has been approved in 2002 for adults and only in 2012 for children [5]. This has inevitably led to extensive off-label use of drugs in children with perinatal HIV-1 infection by the clinicians, who, moreover, have to deal with few available literature data regarding the off-label use to CART therapies.

In a Spanish multicentric study including 318 perinatally HIV-1-infected children and adolescents off-label use of cART involved the $69 \%$ of children [6].

In the present study, data collected from the Italian Register of HIV infection in Paediatrics were retrospectively collected and analysed with the aim to evaluate off label use of cART in perinatally HIV-1 infected children in recent years.

\section{Methods}

\section{Data collection}

The Italian Register for HIV Infection in Paediatrics is a database concerning cases of children with HIV-1 infection, established in 1985 on the initiative of the Immunology Study Group, within the Italian Society of Paediatrics. Data have been recorded since June 1, 1985. Data of children born before this period, about $13 \%$ of the data, have been collected retrospectively [7].

The source of the Register is represented by a network of 106 paediatric clinical centres distributed throughout Italy. These centres, which participate voluntarily, register all children with HIV-1 infection. Two coordination centres are located at the Paediatric Clinic of Infectious Diseases of the Department of Paediatrics of the University of Florence and the Department of Paediatric and Adolescent Sciences of the University of Turin. The Registry includes all children with HIV-1 infection, either born to a mother with HIV-1, or identified at birth or reported later. The data are representative of the entire population of children born to mothers with HIV-1 infection in Italy [7].

Data on mother-child couples are collected using specific reporting and follow-up forms and are processed anonymously, in accordance with the relevant guidelines and regulations.

In particular, basic information regarding the mother include: the mode of infection such as sexual, drug addiction, transfusions, endemic area origin; number of pregnancies; mode of delivery; gestational age and clinical condition of the mother at the time of delivery reported according to the CDC classification system. Since 1989, data concerning the mother include a classification of women treated and untreated, since 1993 there is information on the type of therapy, gestational age at the beginning and at the end of the therapy and since 1994, with the introduction of PACTG 076 protocol, it is also indicated whether the treatment has been administered intrapartum or to the newborn. If the treatment has been administered during pregnancy, all information concerning the combination of drugs used is reported in a special section. Information on possible resistance to treatment, concomitant maternal infections and the conformity of therapy is not collected. The immunological condition of the mother, indicated by the CD4 $4^{+}$lymphocyte count, and the viral load (VL) at the time of delivery have been reported since 1996 and are only known in a minority of cases. The information concerning the child reported in the Register, are the following: birth weight, age at first observation, type and duration of breastfeeding and state of infection. Whereas, in the follow-up records there are information regarding the values of anti-HIV-1 antibodies, viral load, T $\mathrm{CD} 4^{+}$lymphocyte count, stage of disease according to CDC classification criteria, age at diagnosis of AIDS, laboratory investigations, therapy used, date of start and discontinuation of antiretroviral therapy, start and type of Pneumocystis jirovecii pneumonia prophylaxis.

Through the use of reporting forms, the referral physician of each center in the Registry provides to transfer to the two coordination centers basic information regarding those who have acquired HIV-1 infection within 13 years of life or who are 
at risk of becoming infected because they were born to HIV-1 positive mothers; while the follow-up forms are filled in and updated every six months by a paediatrician in charge in each center. In fact, once an infection has been diagnosed, the children are visited every two months. In addition, any changes in clinical and immunological status, together with vaccinations, tests performed and treatment used, are recorded on the follow-up form. Children born to HIV-1 positive mothers who have not acquired the infection are examined at least once a year. Through a double cross-check in the two coordination centers, the quality of the information forwarded is checked. In addition, other control mechanisms are used, while maintaining the anonymity of children, to avoid double reporting. Finally, at least once a year, an audit of the referring physicians is organized in order to verify the results obtained, to plan and encourage research lines and to standardize procedures.

At the moment of patient's enrolment, written informed consent is obtained from a patient's parent or legal guardian; data are treated anonymously as each patient is identified by an alphanumerical code. The study was approved by the review boards and ethics committees of each participating institution; in particular, the ultimate form of the study was approved by the Ethics Committee for Human Investigation at Meyer Children's University Hospital.

\section{Study design}

We performed a cross-sectional study. Children enrolled into the Italian Register for HIV Infection in Paediatrics were selected whether they: have perinatal HIV-1 infection, were receiving CART and were in follow-up at the December 31, 2018. Children were excluded from the study whether they were: uninfected; with horizontally acquired HIV-1 infection (through transfusions, drug addiction, other); not receiving cART at the December 31, 2018, or aged $>18$ years.

The following information has been collected for each patient: age (years); sex (male/female); body weight (Kg); clinical class (class $N, A, B, C$ according to CDC classification); immunological category (category 1, 2, 3 according to CDC classification); $\mathrm{CD}^{+} \mathrm{T}$ lymphocytes count (cell/ $\left.\mu \mathrm{L}\right)$; birthplace of the child and his/her mother (Italian nationality/immigrant), viral load (VL, copies/mL).

The therapy children were receiving was categorized by distinguishing between off-label and on-label use. We have considered off-label use when the drug was prescribed under the age or weight it was licensed for according to the European Medicine Agency (EMA) [5]. Unfortunately, due to a large portion of missing data regarding the children's weight, it was possible to evaluate off-label prescription by weight only relatively to few drugs.

For each therapeutic regimen the following data have been extracted from the Register: type of therapeutic regimen, number of drugs and specific drugs included in the regimen, year in which the therapeutic regimen was started, duration of therapy, age at the beginning of the regimen, clinical status, viral load and count and percentage of CD4 ${ }^{+} T$ lymphocytes at the beginning of therapy and at the last follow-up and the age at the last control and reported adverse events.

Immunological success was defined as a percentage of CD4 ${ }^{+} T$ lymphocytes $>25 \%$ or an increase $>10 \%$ between the beginning of the regimen and the last observation if the regimen was in progress or at the end of the regimen if it was discontinued. When the percentage of $\mathrm{CD}^{+} \mathrm{T}$ lymphocytes at the baseline and the last observations were both $>25 \%$, we considered it as an immunological success. Virological success was defined as a VL not detectable at the last observation during therapy. Due to changes in the detection limits of the HIV RNA test over time, the undetectable VL was defined as $<400$ copies/mL.

\section{Definitions}

\section{Definition of perinatal HIV-1 infection:}

Perinatal infection is defined as the transmission of the infection in utero (congenital infection), during labour, or immediately after birth in breastfed children [8]. 
HIV-1 infection was diagnosed on the basis of the persistence of anti-HIV-1 antibodies after 18 months of life or, before 18 months, by detecting the positivity of viral markers on at least two occasions, excluding any investigations carried out on umbilical cord blood. Children for whom the detection of antibodies to HIV-1 and the PCR investigation were negative on at least two occasions are defined as non-infected [9].

\section{Definition of CART:}

CART was defined as the combination of three or more antiretroviral drugs of at least two different classes [10-11].

\section{Definition of off-label use of drugs in paediatric age:}

Off-label use of a drug was defined as the use of pharmaceutical drugs for an unapproved indication or in an unapproved age group, dosage, or route of administration, according to the package leaflet of medicines authorized by EMA [5]. Detailed data are reported in Appendix, Table 1.

\section{Statistical Analysis}

The continuous variables were expressed as median and interquartile range (IQR). The categorical variables have been expressed as numbers and percentages.

For the categorical variables we performed the $\chi 2$ or Fisher test; for the continuous variables the medians between groups were compared using the Wilcoxon Mann-Whitney $U$ test. Univariate logistic regression analysis was used to evaluate possible risk factors for receiving an off-label therapy, and correspondent odds ratios (ORs) and 95\% confidence intervals were calculated. Statistical analyses were performed using the STATA/SE version 10.0 software package (Stata Corporation, College Station, TX). P $<0.05$ was considered statistically significant.

\section{Results}

Two hundred and twenty-five children were included in the study. Characteristics of the study children are summarized in Table 1

Table 1. Characteristics of the included children $(N=225)$ 


\begin{tabular}{|c|c|c|c|c|c|c|c|c|c|}
\hline \multirow[b]{2}{*}{ All children } & \multicolumn{3}{|c|}{ hildren On Label (n) } & \multicolumn{3}{|c|}{ Children Off-Label (n) } & \multirow{2}{*}{$\begin{array}{l}\text { Total } \\
225\end{array}$} & \multirow{2}{*}{$\begin{array}{l}\% \\
100 \%\end{array}$} & \multirow[t]{2}{*}{$p$ value } \\
\hline & $\begin{array}{c}175 \\
(77.7 \%)\end{array}$ & $\begin{array}{l}\% \text { on } \\
\text { label }\end{array}$ & $\begin{array}{c}\% \\
\text { (within } \\
\text { cathegory) }\end{array}$ & $\begin{array}{c}50 \\
(22.2 \%)\end{array}$ & $\begin{array}{l}\% \text { off } \\
\text { label }\end{array}$ & $\begin{array}{c}\% \text { (within } \\
\text { cathegory) }\end{array}$ & & & \\
\hline \multicolumn{10}{|l|}{ Sex } \\
\hline Males & 81 & $46.2 \%$ & $77.88 \%$ & 23 & $46.0 \%$ & $22.12 \%$ & 104 & $100 \%$ & \\
\hline Females & 94 & $53.8 \%$ & $77.69 \%$ & 27 & $54.0 \%$ & $22.31 \%$ & 121 & $100 \%$ & 0.97 \\
\hline \multicolumn{10}{|l|}{ Age Last Controls } \\
\hline$<1$ year & 0 & $0.0 \%$ & $0.00 \%$ & 2 & $4.0 \%$ & $100.00 \%$ & 2 & $100 \%$ & \\
\hline $1-2$ years & 7 & $4.0 \%$ & $87.50 \%$ & 1 & $2.0 \%$ & $12.50 \%$ & 8 & $100 \%$ & \\
\hline $3-5$ years & 16 & $9.1 \%$ & $84.21 \%$ & 3 & $6.0 \%$ & $15.79 \%$ & 19 & $100 \%$ & \\
\hline $6-11$ years & 47 & $26.9 \%$ & $70.15 \%$ & 20 & $40.0 \%$ & $29.85 \%$ & 67 & $100 \%$ & \\
\hline$>=12$ years & 105 & $60.0 \%$ & $81.40 \%$ & 24 & $48.0 \%$ & $18.60 \%$ & 129 & $100 \%$ & \\
\hline Median age (months; IQR) & \multicolumn{3}{|c|}{$169.13(106-204.9)$} & & \multicolumn{2}{|c|}{137.08 (98.5-181.5) } & \multicolumn{3}{|c|}{$\begin{array}{l}159.33(102.6-201.2) \\
0.0519\end{array}$} \\
\hline
\end{tabular}

\section{Follow-up}

\begin{tabular}{|c|c|c|c|c|c|c|c|c|c|}
\hline Followed since birth & 52 & $29.8 \%$ & $78.79 \%$ & 14 & $28.0 \%$ & $21.21 \%$ & 66 & $100 \%$ & 0.814 \\
\hline \multicolumn{10}{|l|}{ Origin (Child/Mother) } \\
\hline Foreign Country/Missing & 22 & $12.6 \%$ & $91.67 \%$ & 2 & $4.0 \%$ & $8.33 \%$ & 24 & $100 \%$ & \\
\hline Foreign Country/Foreign Country & 54 & $30.9 \%$ & $80.60 \%$ & 13 & $26.0 \%$ & $19.40 \%$ & 67 & $100 \%$ & \\
\hline Italy/Missing & 33 & $18.9 \%$ & $80.49 \%$ & 8 & $16.0 \%$ & $19.51 \%$ & 41 & $100 \%$ & \\
\hline Italy/Foreign Country & 42 & $24.0 \%$ & $76.36 \%$ & 13 & $26.0 \%$ & $23.64 \%$ & 55 & $100 \%$ & \\
\hline Italy/Italy & 24 & $13.8 \%$ & $63.16 \%$ & 14 & $28.0 \%$ & $36.84 \%$ & 38 & $100 \%$ & \\
\hline \multicolumn{10}{|l|}{ Age at the Beginning of cART } \\
\hline$<1$ year & 7 & $4.0 \%$ & $58.33 \%$ & 5 & $10.0 \%$ & $41.67 \%$ & 12 & $100 \%$ & \\
\hline $1-2$ years & 14 & $8.0 \%$ & $56.00 \%$ & 11 & $22.0 \%$ & $44.00 \%$ & 25 & $100 \%$ & \\
\hline $3-5$ years & 23 & $13.1 \%$ & $82.14 \%$ & 5 & $10.0 \%$ & $17.86 \%$ & 28 & $100 \%$ & \\
\hline $6-11$ years & 44 & $25.1 \%$ & $72.13 \%$ & 17 & $34.0 \%$ & $27.87 \%$ & 61 & $100 \%$ & \\
\hline$>=12$ years & 87 & $49.8 \%$ & $87.88 \%$ & 12 & $24.0 \%$ & $12.12 \%$ & 99 & $100 \%$ & \\
\hline Median age (months, IQR) & \multicolumn{3}{|c|}{135 (65.4-184.27) } & & \multicolumn{2}{|c|}{$97.63(17.7-136.7)$} & \multicolumn{3}{|c|}{$\begin{array}{c}126.6(56.3-176.67) \\
0.001\end{array}$} \\
\hline
\end{tabular}

\section{Number of Previous cART}

Regimens

\begin{tabular}{|c|c|c|c|c|c|c|c|c|c|}
\hline 1 regimen & 30 & $17.1 \%$ & $66.67 \%$ & 15 & $30.0 \%$ & $33.33 \%$ & 45 & $100 \%$ & 0.045 \\
\hline 2 regimens & 43 & $24.5 \%$ & $86.00 \%$ & 7 & $14.0 \%$ & $14.00 \%$ & 50 & $100 \%$ & \\
\hline 3 regimens & 28 & $16.0 \%$ & $71.79 \%$ & 11 & $22.0 \%$ & $28.21 \%$ & 39 & $100 \%$ & \\
\hline 4 regimens & 20 & $11.4 \%$ & $74.07 \%$ & 7 & $14.0 \%$ & $25.93 \%$ & 27 & $100 \%$ & \\
\hline 5 regimens & 22 & $12.6 \%$ & $81.48 \%$ & 5 & $10.0 \%$ & $18.52 \%$ & 27 & $100 \%$ & \\
\hline 6 regimens & 16 & $9.1 \%$ & $94.12 \%$ & 1 & $2.0 \%$ & $5.88 \%$ & 17 & $100 \%$ & \\
\hline 7 regimens & 7 & $4.0 \%$ & $77.78 \%$ & 2 & $4.0 \%$ & $22.22 \%$ & 9 & $100 \%$ & \\
\hline 8 regimens & 5 & $2.8 \%$ & 83.33\% & 1 & $2.0 \%$ & $16.67 \%$ & 6 & $100 \%$ & \\
\hline
\end{tabular}




\begin{tabular}{|c|c|c|c|c|c|c|c|c|c|}
\hline 9 regimens & 1 & $0.6 \%$ & $100.00 \%$ & 0 & $0.0 \%$ & $0.00 \%$ & 1 & $100 \%$ & \\
\hline 10 regimens & 1 & $0.6 \%$ & $50.00 \%$ & 1 & $2.0 \%$ & $50.00 \%$ & 2 & $100 \%$ & \\
\hline 11 regimens & 2 & $1.1 \%$ & $100.00 \%$ & 0 & $0.0 \%$ & $0.00 \%$ & 2 & $100 \%$ & \\
\hline \multicolumn{10}{|l|}{ Pre-cART VL } \\
\hline Detectable & 52 & $29.8 \%$ & $68.42 \%$ & 24 & $48.0 \%$ & $31.58 \%$ & 76 & $100 \%$ & 0.021 \\
\hline Undetectable & 68 & $38.9 \%$ & $83.95 \%$ & 13 & $26.0 \%$ & $16.05 \%$ & 81 & $100 \%$ & \\
\hline Missing & 55 & $31.4 \%$ & $80.88 \%$ & 13 & $26.0 \%$ & $19.12 \%$ & 68 & $100 \%$ & \\
\hline \multicolumn{10}{|c|}{ VL at last check } \\
\hline Detectable & 29 & $16.6 \%$ & $74.36 \%$ & 10 & $20.0 \%$ & $25.64 \%$ & 39 & $100 \%$ & 0.583 \\
\hline Undetectable & 145 & $82.9 \%$ & $78.38 \%$ & 40 & $80.0 \%$ & $21.62 \%$ & 185 & $100 \%$ & \\
\hline Missing & 1 & $0.6 \%$ & $100.00 \%$ & 0 & $0.0 \%$ & $0.00 \%$ & 1 & $100 \%$ & \\
\hline \multicolumn{10}{|c|}{ Pre cART CD4 ${ }^{+}$Lymphocytes Count } \\
\hline$<15 \%$ & 12 & $6.9 \%$ & $85.71 \%$ & 2 & $4.0 \%$ & $14.29 \%$ & 14 & $100 \%$ & 0.472 \\
\hline $15 \%-24 \%$ & 15 & $8.6 \%$ & $75.00 \%$ & 5 & $10.0 \%$ & $25.00 \%$ & 20 & $100 \%$ & \\
\hline$>=25 \%$ & 91 & $52.0 \%$ & $77.78 \%$ & 26 & $52.0 \%$ & $22.22 \%$ & 117 & $100 \%$ & \\
\hline Missing & 57 & $32.6 \%$ & $77.03 \%$ & 17 & $34.0 \%$ & $22.97 \%$ & 74 & $100 \%$ & \\
\hline \multicolumn{10}{|c|}{$\begin{array}{l}\text { CD4 }{ }^{+} \text {Lymphocytes Count at last } \\
\text { check }\end{array}$} \\
\hline$<15 \%$ & 4 & $2.3 \%$ & $50.00 \%$ & 4 & $8.0 \%$ & $50.00 \%$ & 8 & $100 \%$ & 0.0543 \\
\hline $15 \%-24 \%$ & 15 & $8.6 \%$ & $93.75 \%$ & 1 & $2.0 \%$ & $6.25 \%$ & 16 & $100 \%$ & \\
\hline$>=25 \%$ & 156 & $89.1 \%$ & $77.61 \%$ & 45 & $90.0 \%$ & $22.39 \%$ & 201 & $100 \%$ & \\
\hline
\end{tabular}

VL: Viral Load. cART: combination antiretroviral therapy

175/225 children (77.7\%) were receiving on-label cART, while 50/225 (22.2\%) were receiving an off-label regimen. At last check, off-label cART was administered to the $100 \%(2 / 2)$ of children aged $<1$ years, $12.5 \%(1 / 8)$ of children aged $1-2$ years, $15.8 \%(3 / 19)$ between $3-5$ years, $29.9 \%$ (20/67) between $6-11$ years, and $18.6 \%(24 / 129)>12$ years.

VL prior of current CART therapy was detectable in $48.0 \%$ (24/50) of off-label patients and in $29.7 \%(52 / 175)$ of on-label patients $(p=0.021$, while no difference among groups was observed ad last check (detectable VL in $20.0 \%$ of children receiving off label cART and in $16.6 \%$ of children on on-label cART; $p=0.583$ ) [Table 1].

Proportions of children with a CD4 ${ }^{+} \mathrm{T}$ lymphocytes percentage $<15 \%$ pre-current $\mathrm{CART}$ and at last check were similar in onlabel and off-label patients [Table 1].

At univariate analysis, risk factors significantly associated with the probability to receive off-label cART were a pre-therapy detectable VL (OR:2.41; 95\%IC 1.13-5.19; $\mathrm{P}=0.024)$, age < 2 years at the beginning of the current regimen (OR:3.24; $95 \%$ IC 1.63-7.30; $P=0.001)$ and being on the first cART regimen (OR:2.07; 95\%IC 1.0006-4.26; $P=0.048$ ) [Table 2]. 
Table 2

Univariate logistic regression analysis

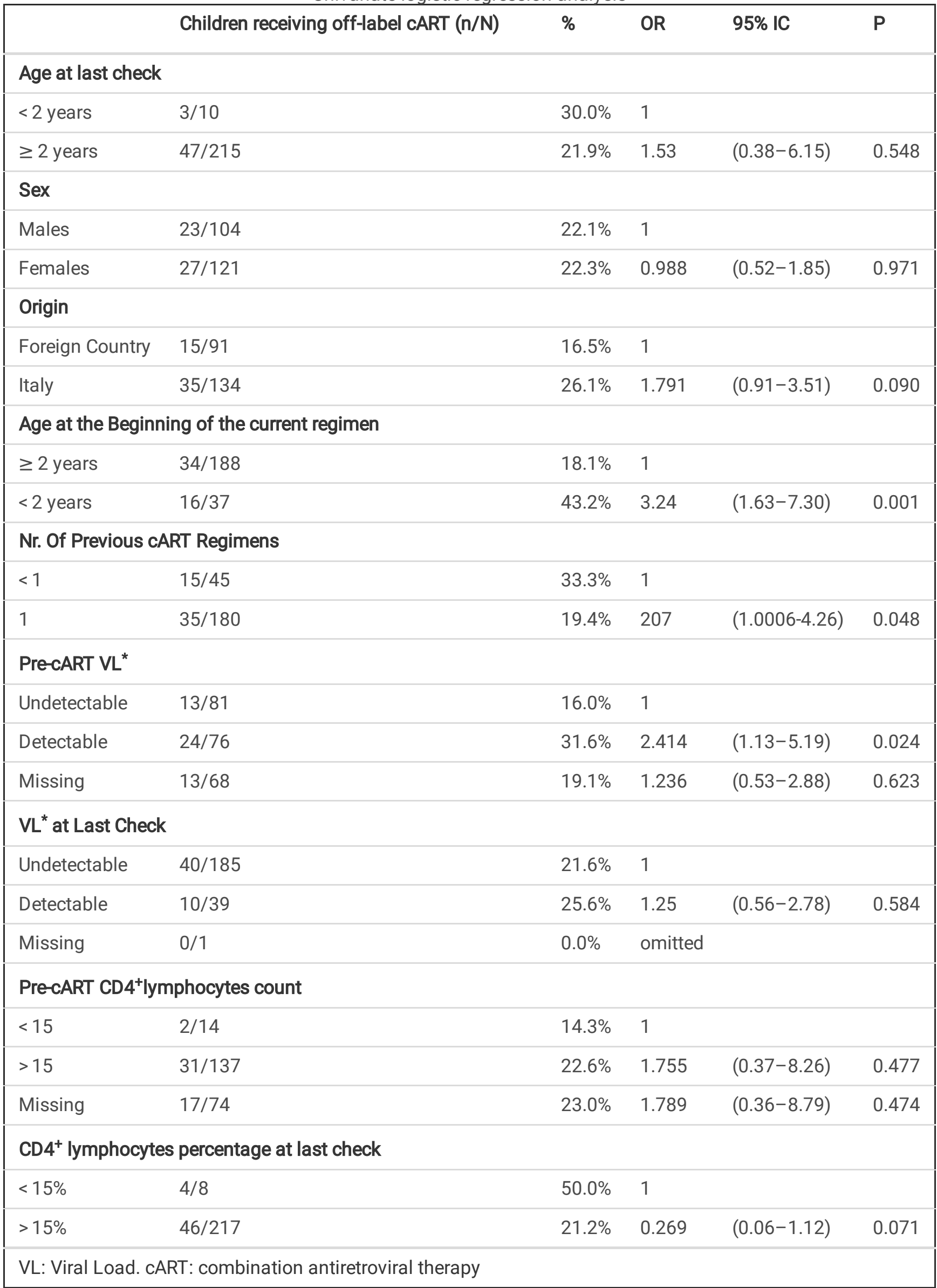


At multivariate analysis age $<2$ years was confirmed to be an independent risk factor for receiving off-label cART (OR:2.75; $95 \% \mathrm{Cl}$ 1.24-6.09; $\mathrm{P}=0.013$ ) [Table 3].

Table 3

Multivariate logistic regression analysis

\begin{tabular}{|llllll|}
\hline \multicolumn{7}{|c|}{ Children receiving off-label cART $(\mathrm{n} / \mathrm{N})$} & $\%$ & OR & $95 \%$ IC & P \\
\hline \multicolumn{7}{|l|}{ Age at the Beginning of the current regimen } & & & & \\
\hline$\geq 2$ years & $34 / 188$ & $18.1 \%$ & 1 & & \\
\hline$<2$ years & $16 / 37$ & $43.2 \%$ & 2.75 & $(1.24-6.09)$ & 0.013 \\
\hline Pre-cART VL & & & & & \\
\hline Undetectable & $13 / 81$ & $16.0 \%$ & 1 & & \\
\hline Detectable & $24 / 76$ & $31.6 \%$ & 2.078 & $(0.95-4.56)$ & 0.068 \\
\hline Missing & $13 / 68$ & $19.1 \%$ & 1.103 & $(0.47-2.61)$ & 0.824 \\
\hline VL: Viral Load. cART: combination antiretroviral therapy & & & & \\
\hline
\end{tabular}

\section{Sub-analysis of most commonly drugs prescribed off-label}

The most commonly off-label drugs administered were: lopinavir/ritonavir (10/56, 17.9\%) tenofovir disoproxil/emtricitabine $(11 / 11,100 \%)$, dolutegravir/abacavir/lamivudine (8/26, 30.8\%), elivitergravir/cobicistat/emtricitabine/tenofovir alafenamide (5/11, 45.5\%) [Table 4]. 
Percentages of off-label administration by age and weight of each drug

\begin{tabular}{|c|c|c|c|c|c|c|c|c|}
\hline & \multicolumn{5}{|c|}{ Off-label drugs by age } & \multicolumn{3}{|c|}{$\begin{array}{l}\text { Off-label drugs by } \\
\text { weight }\end{array}$} \\
\hline & $\begin{array}{l}\text { ON } \\
\text { LABEL }\end{array}$ & $\begin{array}{l}\text { OFF } \\
\text { LABEL }\end{array}$ & Tot & $\begin{array}{l}\% \text { Off } \\
\text { Label } \\
\text { Age }\end{array}$ & $\begin{array}{l}\text { ON } \\
\text { LABEL }\end{array}$ & $\begin{array}{l}\text { OFF } \\
\text { LABEL }\end{array}$ & Missing & Tot \\
\hline \multicolumn{9}{|c|}{$\begin{array}{l}\text { Nucleoside Reverse Transcriptase Inhibitors } \\
\text { (NRTI) }\end{array}$} \\
\hline Abacavir (ABC) & 35 & 1 & 36 & $2.8 \%$ & 26 & 0 & 10 & 36 \\
\hline Didanosina (ddl) & 1 & 0 & 1 & $0.0 \%$ & 0 & 0 & 1 & 1 \\
\hline Emtricitabina (FTC) & 4 & 0 & 4 & $0.0 \%$ & 2 & 0 & 2 & 4 \\
\hline Lamivudina (3TC) & 76 & 5 & 81 & $6.2 \%$ & 44 & 0 & 37 & 81 \\
\hline Tenofovir (TDF) & 4 & 0 & 4 & $0.0 \%$ & 3 & 0 & 1 & 4 \\
\hline Zidovudina (ZDV) & 26 & 0 & 26 & $0.0 \%$ & 15 & 0 & 11 & 26 \\
\hline Tenofovir alafenamide (TAF) & 3 & 0 & 3 & $0.0 \%$ & 1 & 0 & 2 & 3 \\
\hline \multicolumn{9}{|l|}{$\begin{array}{l}\text { Non-nucleoside reverse transcriptase } \\
\text { inhibitors (NNRTI) }\end{array}$} \\
\hline Efavirenz (EFV) & 8 & 1 & 9 & $11.1 \%$ & 4 & 0 & 5 & 9 \\
\hline Etravirina (EVR) & 3 & 2 & 5 & $40.0 \%$ & 3 & 2 & 0 & 5 \\
\hline Nevirapina (NVP) & 19 & 0 & 19 & $0.0 \%$ & 11 & 0 & 8 & 19 \\
\hline Rilpivirina (RPV) & 1 & 0 & 1 & $0.0 \%$ & 0 & 0 & 1 & 1 \\
\hline \multicolumn{9}{|l|}{ Protease inhibitors (PI) } \\
\hline Atazanavir (ATV) & 2 & 2 & 4 & $50.0 \%$ & 3 & 0 & 1 & 4 \\
\hline Darunavir (DRV) & 46 & 4 & 50 & $8.0 \%$ & 30 & 1 & 19 & 50 \\
\hline Ritonavir (RTV) & 51 & 1 & 52 & $1.9 \%$ & 32 & 0 & 20 & 52 \\
\hline \multicolumn{9}{|l|}{ Integrase inhibitors (INSTI) } \\
\hline Raltegravir (RAL) & 21 & 1 & 22 & $4.5 \%$ & 20 & 0 & 2 & 22 \\
\hline Dolutegravir (DTG) & 18 & 0 & 18 & $0.0 \%$ & 7 & 0 & 11 & 18 \\
\hline Elvitegravir (EVG) & 2 & 0 & 2 & $0.0 \%$ & 2 & 0 & 0 & 2 \\
\hline \multicolumn{9}{|l|}{ Booster } \\
\hline Cobicistat & 1 & 0 & 1 & $0.0 \%$ & 1 & 0 & 0 & 1 \\
\hline \multicolumn{9}{|l|}{ Fixed-dose combined drugs } \\
\hline Lopinavir/ritonavir & 46 & 10 & 56 & $17.9 \%$ & 39 & 0 & 17 & 56 \\
\hline Zidovudine/lamivudine & 7 & 1 & 8 & $12.5 \%$ & 5 & 1 & 2 & 8 \\
\hline Lamivudine/abacavir & 30 & 0 & 30 & $0.0 \%$ & 18 & 3 & 9 & 30 \\
\hline Tenofovir/emtricabine & 0 & 11 & 11 & $100.0 \%$ & 6 & 5 & 0 & 11 \\
\hline Emtricitabine/tenofovir/efavirenz & 0 & 1 & 1 & $100.0 \%$ & 1 & 0 & 0 & 1 \\
\hline
\end{tabular}




\begin{tabular}{|c|c|c|c|c|c|c|c|c|}
\hline \multirow[b]{2}{*}{ Emtricitabina/rilpivirina/tenofovir } & \multirow[b]{2}{*}{0} & \multicolumn{4}{|c|}{ Off-label drugs by age } & \multicolumn{3}{|c|}{$\begin{array}{l}\text { Off-label drugs by } \\
\text { weight }\end{array}$} \\
\hline & & 1 & 1 & $100.0 \%$ & 1 & 0 & 0 & 1 \\
\hline Dolutegravir/abacavir/lamivudine & 18 & 8 & 26 & $30.8 \%$ & 15 & 5 & 6 & 26 \\
\hline Darunavir/cobicistat & 11 & 0 & 11 & $0.0 \%$ & 4 & 0 & 7 & 11 \\
\hline Tenofovir alafenamide/emtricitabine & 15 & 2 & 17 & $11.8 \%$ & 11 & 1 & 5 & 17 \\
\hline $\begin{array}{l}\text { Elvitegravir/cobicistat/emtricitabine/tenofovir } \\
\text { alafenamide }\end{array}$ & 6 & 5 & 11 & $45.5 \%$ & 7 & 4 & 0 & 11 \\
\hline $\begin{array}{l}\text { Emtricitabine/rilpivirine/tenofovir } \\
\text { alafenamide }\end{array}$ & 7 & 0 & 7 & $0.0 \%$ & 3 & 0 & 4 & 7 \\
\hline $\begin{array}{l}\text { Darunavir/cobicistat/emtricitabine/tenofovir } \\
\text { alafenamide }\end{array}$ & 5 & 0 & 5 & $0.0 \%$ & 5 & 0 & 0 & 5 \\
\hline
\end{tabular}

VL at last check, in patients treated with lopinavir/ritonavir, was undetectable in 90\% (9/10) of off-label patients and in $82.2 \%$ $(37 / 45)$ of on-label patients $(p=0.547)$, whereas the last CD4 ${ }^{+}$T lymphocytes percentage was > $25 \%$ in $100 \%(10 / 10)$ of offlabel patients and $91.1 \%(41 / 45)$ of on-label patients $(p=0.983)$ [Appendix, Table 2].

Regarding emtricitabine/tenofovir disoproxil, at last check VL was undetectable in $81.8 \%(9 / 11)$ of off-label patients, whereas the last $C D 4^{+} \mathrm{T}$ lymphocytes percentage was $>25 \%$ in $81.8 \%(9 / 11)$ of off-label patients. We also observed that no child received on-label treatment with this drug [Appendix, Table 3].

Regarding elvitegravir/cobicistat/emtricitabine/tenofovir alafenamide, at last check the VL was undetectable in 100\% (5/5) of off-label patients and in $100 \%(6 / 6)$ of on-label patients, whereas the last CD $4^{+}$T lymphocytes percentage was $>25 \%$ in $100 \%$ $(5 / 5)$ of off-label patients and in $100 \%(6 / 6)$ of on-label patients [Appendix, Table 4].

Finally, at last check VL in patients on therapy with abacavir/dolutegravir/lamivudine was undetectable in $87.5 \%(7 / 8)$ of offlabel patients and in $77.7 \%(14 / 18)$ of on-label patients $(p=0.561)$, whereas the last CD $4^{+} \mathrm{T}$ lymphocytes percentage was > $25 \%$ in $87.5 \%(7 / 8)$ of off-label patients and in $94.4 \%(17 / 18)$ of on label patients $(p=0.539)$ [Appendix, Table 5].

\section{Discussion}

Off-label drugs in our cohort of perinatally HIV-1 infected children were prescribed to $22.2 \%(50 / 225)$ of children at their last check in 2018. At univariate logistic regression analysis, risk factors for receiving an off-label regimen were age $<2$ years, being of first HAART regimen and a detectable VL before starting that regimen. At multivariate analysis only younger age was confirmed to be an independent risk factor for receiving an off-label therapy. However, we also noticed that several children $(56.52 \%, 13 / 23)$, aged between 11 and 12 years of age were receiving an off-label cART at last check. In this sub-group of children physicians probably considered minimal the potential risks related to an off-label use of the ARV drug since 12 years was the age for which the drug was licensed.

The most commonly antiretroviral drugs prescribed off -label were lopinavir-ritonavir (Kaletra $\left.{ }^{\circledR}\right)$ in younger children, and three fixed dose combinations in older children: elvitegravir/cobicistat/emtricitabine/tenofovir alafenamide (Genvoya $\left.{ }^{\circledR}\right)$, abacavir/dolutegravir/lamivudine (Triumeq $\left.{ }^{\circledR}\right)$ and tenofovir disoproxil/emtricitabine (Truvada $\left.{ }^{\circledR}\right)$.

Comparing our results to a 2016 Spanish multicentre study, conducted by Cooke et al. on a cohort of 318 perinatally HIV-1infected children and adolescents between 1988 and 2012, it was found that our off-label therapy rates in children are lower than the ones of the Spanish sample, which reveals an off-label use of antiretroviral therapy in $69 \%$ of included children $(221 / 318)$ [6]. This difference could reflect the increase in approval coverage for the clusters under 2 years of age, which 
occurred after 2012 and consequently reduced the off-label incidence. For example, in 2012 atazanavir was approved in children over 6 years of age, while in 2014 the approval was brought forward to children over 3 months of age; raltegravir in 2012 was approved only in children over 2 years of age, while by 2018 it was approved from birth; efavirenz, which until 2013 was approved only for children over 3 years of age, is now approved in children over 3 months of age [5]. Considering that efavirenz was used by the $24.5 \%$ of our population and that among the non-nucleoside reverse transcriptase inhibitors (NNRTIs) it was the most widely used after nevirapine, this fact may have contributed to reduce the off-label percentage in our population. However, in our cohort, off-label use was still widespread. The $10 \%$ of our off-label patients have reached the fifth therapeutic regimen; this confirms that off-label therapies are chosen in some multi-experienced children, failing previous therapy and probably with no in-label therapeutic options available.

In our study, before the current cART, the $48 \%$ of the off-label children had a detectable VL, while, at the last check, the $80 \%$ of the patients had an undetectable VL and the $90 \%$ a CD4 $4^{+}$lymphocyteS count $>25 \%$. Similar to our results, Cooke et al. found that the main reason for the off-label use of CART was the virological failure of the previous therapy. Moreover, the onset of adverse events and problems related to the administration of drugs, such as formulations unsuitable for age, respectively led in $12 \%$ and $5 \%$ of cases to the discontinuation of treatment. Adherence to the therapy is essential for its effectiveness and this is in agreement with our data, since the main drugs used off-label are fixed-combination drugs. Clay et al. meta-analysis, comparing adult patients' adherence of single tablet regimens (STR) to multiple tablet regimens (MTR), revealed that patients on STR cART were significantly more adherent and had a better VL suppression when compared to patients on MTR [12]. Indeed, in our study, we observed that four MTR (lopinavir/ritonavir; emtricitabine/tenofovir disoproxil; elvitegravir/cobicistat/emtricitabine/tenofovir alafenamide and abacavir/dolutegravir/lamivudine) were the most commonly used off-label drug formulations.

Since 2012 lopinavir/ritonavir is licensed for the use in children aged > 14 days, while since 2002 it was licensed for children aged $>2$ years. In our dataset, the age group receiving off-label lopinavir/ritonavir was mostly represented by children aged < 12 months. Previous studies found that lopinavir/ritonavir is more effective than nevirapine, which may be one of the reasons why physician decided to start this therapy [13]. However, since cases of lopinavir/ritonavir cardiotoxicity (complete heart block and cardiomyopathy) have been reported in infants, caution is recommended when using this drug [13]. No cardiac adverse event in young children was reported in our dataset. What we can observe instead, is a good therapeutic success, demonstrated by the fact that $100 \%$ of children receiving off label lopinavir/ritonavir had a CD $4^{+} \mathrm{T}$ lymphocytes percentage at last check-up $>25 \%$ and $90 \%$ had an undetectable viremia.

For what concerns elvitegravir/cobicistat/emtricitabine/tenofovir alafenamide, the age most involved by their off-label use was 8-11 years; in our cohort, $100 \%$ of off-label patients the last VL was undetectable and the CD $4^{+} \mathrm{T}$ lymphocytes percentage $>25 \%$. In the same age group, we also find off-label use for abacavir/dolutegravir/lamivudine. In these children an undetectable $\mathrm{VL}$ at the last check was reached in the $87.7 \%$ of patients and undetectable and $\mathrm{CD} 4^{+} \mathrm{T}$ lymphocytes percentage $>25 \%$ in the $87.5 \%$ of them.

Regarding emtricitabine/tenofovir disoproxil, the age most involved by off-label use was 11-14 years, (the drug has to be considered off label when used in children aged 14 years since when these children started the therapy it was approved $>18$ years). At the last check the VL was undetectable in the $81.8 \%$ and $C D 4^{+} \mathrm{T}$ lymphocytes percentage $>25 \%$ in the $81.8 \%$.

In our sample, a 10 years old child received an off-label treatment with rilpivirine, emtricitabine and tenofovir disoproxil. Before the current therapy the patient had a detectable viral load, while at the last check the VL was undetectable. No adverse event was reported for this patient. A previous multicentre study that enrolled 17 children and adolescents with perinatal HIV1 infection from 2013 to 2015 demonstrated the efficacy of off-label use of rilpivirine in combination with emtricitabine and tenofovir disoproxil, as children showed good control of the infection, improved $\mathrm{CD} 4^{+} \mathrm{T}$ lymphocytes count and $\mathrm{CD} 4^{+} / \mathrm{CD} 8^{+} \mathrm{T}$ lymphocytes ratio [14]. 
Our data suggest similar proportion of virological and immunological success at last check among children receiving off- or on-label cART. No adverse event to off-label ARV drug was reported in our dataset.

The potential limitations of our study lie in its retrospective design. In addition, even if no adverse event related to the use of off-label ARV drug was reported we may not exclude an underreporting bias. Finally, we could not explore the risk of prescription of not-corrected dose in children receiving an off-label regimen, due to a large proportion of missing data regarding body weights.

In a previous study more than $10 \%$ of children treated with an off-label prescription received an overdose and a further $10 \%$ received an underdose, defined as the administration of a $25 \%$ dose respectively above or below the recommended dose [6]. A previous multi-centre cohort study from Ireland and UK, including 615 children aged 2-12 years and treated with cART, showed that children receiving a dose less than $90 \%$ of the recommended dose accounted for $6-62 \%$ of the study population [15].

\section{Conclusions}

The prescription of an off-label cART in perinatally HIV-1 infected children is common, in particular in children with detectable VL despite previous therapies. Our data suggest similar proportion of virological and immunological success at last check among children receiving off- or on-label cART. Even if our safety data regarding off-label regimens where poor, no adverse event was reported in our dataset. However, larger studies are needed to better clarified efficacy and safety of off-label cART regimens in children.

In our dataset, the prescription of an off-label cART was more common in children aged $<2$ years, likely due to the fact that only the $34.4 \%$ of ARVs are approved for use in this age group. Moreover, we could assume that in several cases the physician choice to undertake an off-label therapy was probably dictated by a virological failure with previous regimens, possibly due to the presence of drug resistance viral strains or poor patient adherence.

Efforts should be made in order to allow an on-label prescription in HIV-1 infected children.

\section{Abbreviations}

HAART

highly active antiretroviral therapy;

EMA

European Medicine Agency;

$\mathrm{VL}$

viral load;

ARV

antiretroviral;

CART

combination antiretroviral therapy;

IQR

interquartile range;

NNRTIs

non-nucleoside reverse transcriptase inhibitors

STR

single tablet regimens;

MTR

multiple tablet regimens. 


\section{Declarations}

\section{Ethics approval and consent to participate}

At the moment of patient's enrolment, written informed consent was obtained from a patient's parent or legal guardian; data are treated anonymously as each patient is identified by an alphanumerical code. Data regarding mother-child pairs are collected using specific individual forms for registration and follow-up.

The study was approved by the review boards and ethics committees of each participating institution; in particular, the ultimate form of the study was approved by the Ethics Committee for Human Investigation at Meyer Children's University Hospital.

\section{Consent for publication}

Not applicable.

\section{Availability of data and materials}

The datasets used and analysed during the current study are available from the corresponding author on reasonable request.

\section{Competing interests}

Authors declare no conflict of interest relative to the topic of this article. No financial or nonfinancial benefits have been received or will be received from any party related directly or indirectly to the subject of this article.

\section{Funding}

This research did not receive any specific grant from funding agencies in the public, commercial, or not-for-profit sectors.

\section{Authors' contributions}

All Authors and Co-Authors contributed equally to the study design, acquisition of women and children information and interpretation of data. EC and LG drafted the manuscript. All Authors and Co-Authors accepted the final version of the manuscript.

\section{Acknowledgements}

All principal investigators and sites who participate in the "Italian Register for HIV infection in children" are listed below. Maurizio Ruggeri (Bergamo), Francesco Baldi (Imola), Giacomo Faldella (Bologna), Piergiorgio Chiriacò (Brindisi) Carlo Dessì (Cagliari), Maria Grazia Pantò (Catania), Elisa Anastasio (Catanzaro), Luisa Abbagnato (Como), Maria Rita Govoni (Ferrara), Maurizio Bigi (Forli), Elisabetta Bondi (Genova), Riccardo Borea, Giovanni Cenderello (Imperia), Donato Tommasi (Lecce), Raffaella Pinzani (Milano) Ernesto Renato Dalle Nogare, Marcello Saitta (Palermo), Leonardo Felici (Pesaro), Rita Consolini (Pisa), Angelo Antonellini (Ravenna), Gianfranco Anzidei, Orazio Genovese, Salvatore Catania, Fabio Natale (Roma), Paolina Olmeo (Sassari) Letizia Cristiano (Taranto), Vincenzo Portelli (Trapani), Marco Rabusin (Trieste), Sara Parigi (Firenze), Francesca Orlandi (Firenze), Alessandra de Martino (Firenze), Giada Maria DiPietro (Milano), Leone Fabrizio (Roma).

\section{References}

1. Broder S. The development of antiretroviral therapy and its impact on the HV1/AIDS pandemic. Antiviral Res. 2010;85 $1: 1-18$.

2. Clarke DF, Penazzato M, Capparelli E. Prevention and treatment of HIV infection in neonates: evidence base for existing WHO dosing recommendations and implementation considerations. Expert Rev Clin Pharmacol 2018;11:83-93. 
3. Linakis MW, Roberts JK, Lala AC. Challenges associated with route of administration in neonatal drug delivery. Clin Pharmacokinet 2016;55:185-196.

4. Panel on Antiretroviral Guidelines for Adults and Adolescents. Guidelines for the Use of Antiretroviral Agents in Adults and Adolescents with HIV. Department of Health and Human Services. Available at https://clinicalinfo.hiv.gov/sites/default/files/inline-files/AdultandAdolescentGL.pdf. Accessed 20 July 2020.

5. European Medicine Agency. Agency's latest information on HIV and AIDS, available at https://www.ema.europa.eu/en/news-events/therapeutic-areas-latest-updates/hiv-aids. Accessed 20 July 2020.

6. Cooke F E, Rojas P, Holguin A, Gonzalez Tome MI, Jimenez de Ory S, Mellado MJ, Navarro ML, Rojo P, Ramos JT. Description and consequences of prescribing off-label antiretrovirals in the Madrid Cohort of HIV-infected children over a quarter of century (1988-2012). Antiviral Therapy 2106; 21:65-70.

7. Chiappini E, Galli L, Lisi C, Gabiano C, et al. Italian Register for HIV Infection in Children. Risk of perinatal HIV infection in infants born in Italy to immigrant mothers. Clin Infect Dis 2011;53:310-3

8. TremoladaS, Delbue S, Ferrante P. Infections of the fetus and newborn infant. Pediatr Med Chir. 2008;30:177-191

9. Chiappini E, Galli L, Lisi C, Gabiano C, et al. Strategies for prevention of mother-to-child trasmission adopted in the "real word" setting: data from the italian register for HIV-1 infection in children. J Acquir Immune Defic Sindr 2018; 79:54-61

10. Lu DY, Wu HY, Yarla NS, Xu B, Ding J, Lu TR. HAART in HIV/AIDS Treatments: Future Trends. Infect Disord Drug Targets. 2018;18:15-22.

11. Panel on Treatment of Pregnant Women with HIV Infection and Prevention of Perinatal Transmission. Recommendations for the Use of Antiretroviral Drugs in Pregnant Women with HIV Infection and Interventions to Reduce Perinatal HIV Transmission in the United States. Available at https://clinicalinfo.hiv.gov/sites/default/files/inline-files/PerinatalGL.pdf Accessed 20 July 2020.

12. Clay PG, Nag S, Graham CM, Narayanan S. Meta-Analysis of Studies Comparing Single and Multi-Tablet Fixed Dose Combination HIV Treatment Regimens. Medicine (Baltimore). 2015;94:e1677.

13. Violari A, Lindsey JC, Hughes MD. Nevirapine versus ritornavir-boosted lopinavir for HIV-infected children. N Engl J Med. 2012, 366:2380-2389.

14. Falcon-Neyra L, Palladino C, Navarro Gómez ML et al. Off-label use of rilpivirine in combination with emtricitabine and tenofovir in HIV-1-infected paediatric patients A multicenter study. Medicine (Baltimore), 2016; 95: e3842.

15. Menson ES, Walker AS, Sharland M, Wells C, Tudor-Williams G, Riordan FA, Lyall EGH, Mgibb D. Underdosing of antiretrovirals in UK and Irish children with HIV as an example of problems in prescribing medicines to children, 19972005: cohort study. BMJ. 2006; 332:1183-1187.

\section{Supplementary Files}

This is a list of supplementary files associated with this preprint. Click to download.

- Appendix.docx 